\title{
Conhecimento e adesão como fatores associados a acidentes com agulhas contaminadas com material biológico: Brasil e Colômbia
}

Ehideé Isabel Gómez La-Rotta (https://orcid.org/0000-0003-1194-9898) ${ }^{1}$

Clerison Stelvio Garcia (https://orcid.org/0000-0003-0008-5481) ${ }^{1}$

Carlos Morales Pertuz (https://orcid.org/0000-0002-6453-3960) ${ }^{2}$

Isabella de Oliveira Campos Miquilin (https://orcid.org/0000-0003-4196-5901) ${ }^{1}$

Agnes Raquel Camisão (https://orcid.org/0000-0002-7069-6387) ${ }^{3}$

Danilo Donizetti Trevisan (https://orcid.org/0000-0002-6998-9166) ${ }^{4}$

Francisco Hideo Aoki (https://orcid.org/0000-0002-9003-8488) ${ }^{1}$

Heleno Rodrigues Correa-Filho (https://orcid.org/0000-0001-8056-8824) ${ }^{5}$
${ }^{1}$ Faculdade de Ciências Médicas, Universidade Estadual de Campinas. Cidade Universitária Zeferino Vaz, Barão Geraldo. 13083-970 Campinas

SP Brasil.larottaehidee@ gmail.com

${ }^{2}$ Clínica El Country. Bogotá

Colombia.

${ }^{3}$ Curso de Enfermagem, Faculdade Evangélica de Goianésia. Goianésia GO Brasil.

${ }^{4}$ Universidade Federal de São João do Rei. Divinópolis MG Brasil.

${ }^{5}$ Departamento de Saúde Coletiva, Universidade de Brasília. Brasília DF Brasil.
Resumo Fizemos estudo transversal para iniciar coorte em dois Hospitais Universitários de dois países - Brasil e Colômbia - para avaliar a prevalência de acidentes com material biológico (AT-MB), $o$ nível de adesão às Precauções Padrão $(P P)$ e o conhecimento sobre patógenos transmissíveis pelo sangue e fatores associados entre trabalhadores $e$ estudantes da saúde, no marco da implementação da norma NR-32. Criamos escalas para estimar conhecimento e adesão baseadas em 12 e 11 perguntas respectivamente. Utilizamos Regressão de Poisson-Tweedie para avaliar a associação do conhecimento e da adesão às PP com sofrer AT -MB. Avaliamos 965 indivíduos (348 estudantes e 617 profissionais). O conhecimento teve média de 10,98 com mediana de $11(10,12)$ e $\alpha$-Cr de 0,625 . A média de adesão foi de 30,74 com mediana de $31(28,34)$ e $\alpha$-Cr de 0,745 , associandose a País, grupo (estudantes) e percepção de risco. Entre os fatores associadas ao relato de $A T-M B$ encontraram-se o conhecimento, a adesão às $P P$, País de origem e ter tomado o esquema completo de vacinação contra Hepatites B. Concluímos que o nível de conhecimento e adesão foram adequados, ainda melhores entre os participantes do Brasil e associaram-se ao relato $A T-M B$.

Palavras-chave Conhecimento, Adesão as diretrizes, Acidentes de trabalho, Percepção, Risco 


\section{Introdução}

A proporção global de trabalhadores da saúde em relação à população geral é de $0,6 \%$, estimandose cerca de 35,7 milhões de trabalhadores; deles 3 milhões anualmente sofrem exposição a patógenos sanguíneos ${ }^{1}$. Calcula-se que $37,6 \%$ das Hepatites B, 39\% das Hepatites Ce 4,4\% do VIH/SIDA nos Trabalhadores da Saúde em todo o mundo se devem a acidentes com agulhas contaminadas com material biológico ${ }^{1}$.

$\mathrm{Na}$ Colômbia, dados do Ministério do Trabalho informam que $7,51 \%$ dos trabalhadores afiliados ao sistema sofreram algum tipo de acidente de trabalho em 2015. Em relação aos acidentes de trabalho com material biológico (AT-MB), estudos realizados com população afiliada às Administradoras de Riscos Laborais (ARLs) reportam que as atividades relacionadas com o setor saúde ocupam o quinto lugar comparadas com outros setores econômicos com coeficiente de incidência de 6,61 por 100 trabalhadores². $\mathrm{Na} \mathrm{Co}-$ lômbia não se dispõe de dados consolidados sobre o número de AT-MB, nem se conta com um sistema de informação de vigilância que permita quantificar e caracterizar este tipo de acidentes de trabalho ${ }^{3}$.

No Brasil, em 2015, foram registrados no Instituto Nacional de Seguridade Social (INSS) 612,6 mil acidentes de trabalho por todas as causas, com redução de $13,99 \%$ comparada a $2014^{4}$. Na distribuição por atividade econômica os subsetores com maior participação foram 'Saúde e serviços sociais' com 14,49\% e 'Comercio e reparação de veículos automotores' com 13,27\% do total $^{4}$.

$\mathrm{O}$ coeficiente de incidência dos AT-MB no Brasil sofreu aumento. Segundo dados do INSS e do Departamento Intersindical de Estatística e Estudos Socioeconômicos (DIEESE), o número de acidentes/número de trabalhadores expostos $^{\star} 1000$ passou de 2,7 AT-MB em 2004 para 7,6 em $2014^{4,5}$.

Neste panorama, o Brasil foi o primeiro país na America Latina e Caribe a criar uma norma (NR-32 - Seguridade e Saúde no Trabalho em Serviços de Saúde) com o objetivo de minimizar os riscos e proporcionar um ambiente de trabalho mais saudável para os trabalhadores da área da saúde ${ }^{6}$, e consequentemente reduzir doenças infecciosas pela exposição a material biológico entre estes profissionais, por meio de recomendações sobre o uso, descarte e implantação dos dispositivos de segurança para os perfurocortantes ${ }^{7}$. A Colômbia não conta com uma norma específica para os trabalhadores da saúde que tenha adesão igual à do Brasil, às recomendações do Centro de Controle e Prevenção de Doenças (CDC) e às diretrizes da Organização Internacional do Trabalho (OIT).

As recomendações (precauções padrão - PPs) do CDC foram criadas depois da epidemia do HIV/AIDS e publicadas em 1985, com atualização de 1987, que incluía um guia sobre a prevenção de acidentes com perfurocortantes com foco nos cuidados durante o manuseio e o descarte. Estas indicações eram destinadas aos profissionais da saúde e tinham o objetivo de diminuir o risco de contaminação pelos vírus HIV, HBV e HCV, pelo contato com sangue e secreções corporais ${ }^{8}$.

O cumprimento destas recomendações (PPs) requer atitudes apropriadas dos profissionais da saúde durante longos períodos de tempo, exigindo motivação e conhecimento técnico9. Internacionalmente, é considerada uma forma efetiva de proteger os profissionais, os pacientes e o público ${ }^{9}$, além de reduzir as infecções hospitalares $^{8}$. O não cumprimento pode refletir em elevadas taxas de incidência dos AT-MB ${ }^{10}$.

Entre outros fatores associados à incidência de AT-MB, segundo a literatura, encontramos o conhecimento ${ }^{11}$ e a percepção de risco ${ }^{12}$. O conceito de percepção é derivado da descrição dada por Dela Coleta (1986) e adaptado por CorreaFilho (1994) com base nos delineamentos de Breilh (1990), que descreveu percepção como um contravalor, que pode se expressar nos ambientes de trabalho como o relato do registro na memória e nos sentimentos pessoais sobre a relação entre condições de vida, saúde e trabalho e os contravalores ligados à causação ou condições mediadoras de acidentes ou agravos de saúde no ambiente de trabalho ${ }^{13-15}$.

Com estes dados procuramos avaliar a prevalência de AT-MB, o nível de adesão às Precauções Padrão e de conhecimento sobre a transmissão de patógenos transmissíveis pelo sangue entre trabalhadores e estudantes da saúde em dois Hospitais Universitários Brasil-Colômbia, no marco da implementação da NR-32. Perguntamos também se o conhecimento, a adesão e a percepção de risco são fatores predictores de AT-MB.

No Hospital, o tema tem sua importância reforçada por tratar-se de instituição acadêmica, onde os estudantes da área da saúde e residentes aprendem a trabalhar nas melhores condições e adquirem tanto conhecimento como atitudes; o que conseguem ao observarem seus professores, que devem ser exemplo, proporcionando informações e atos médicos de alto nível de excelência ${ }^{16}$. 


\section{Método}

Realizamos estudo transversal para avaliar o nível de adesão e conhecimento entre estudantes da saúde (medicina, enfermagem e odontologia) e trabalhadores da saúde (médicos e enfermeiros) de dois Hospitais Universitários Brasil-Colômbia entre janeiro de 2014 e fevereiro de 2015.

Este artigo reflete o primeiro contato realizado quando se constituiu a coorte de participantes a serem seguidos por um ano para determinar a incidência de acidentes com material biológico, comparando o Brasil, que está implementando a NR-32, com a Colômbia.

Depois de estimar proporções de conhecimento e adesão às precauções padrão de $35 \%{ }^{11,17}$ estabelecemos um erro amostral máximo aceitável de $0,05(5 \%)$ e poder do teste (1-beta) de $80 \%$; calculamos uma amostra binacional total de $n=1252$ participantes, resultantes da soma de 313 para cada grupo de estudantes e profissionais, totalizando 626 em cada País.

A seleção da amostra foi aleatória mediante amostragem sistemática estratificada seguindo a lista de estudantes e profissionais. Incluímos no estudo os participantes que cumpriram com os seguintes critérios: ser profissional da saúde trabalhando há mais de três meses nos Hospitais de estudo no momento da entrevista, que fosse estudante matriculado em alguma das faculdades inclusas no estudo, que pertenciam às instituições responsáveis pelos Hospitais Universitários e/ou que adotaram como campo de pratica os Hospitais estudados.

Para realizar o estudo elaboramos um questionário estruturado (autopreenchível) com sete seções, entre as quais encontravam-se as perguntas de conhecimento sobre meios de transmissão dos vírus das Hepatites B e C e do HIV e de adesão às PPs. Incluímos perguntas sobre hábitos sexuais (número de parceiros sexuais no último ano e uso de proteção) e percepção de risco ocupacional. O questionário foi validado com estudo piloto anterior nos dois países com a finalidade de avaliar e ajustar o construto nos dois idiomas $^{18}$.

A escala de conhecimento continha 12 perguntas com escore entre zero (nenhuma resposta correta) e 12 (todas as respostas corretas). A escala de adesão estava constituída por 11 perguntas com escores totais de zero a 37 pontos.

Recodificamos a variável Hábito Sexual, criando nova variável nomeada comportamento sexual de risco. Atribuirmos 0 há não ter risco e 1 à presença de risco. Na categoria "sem risco" foram incluídos os participantes que no último ano no tiveram relações sexuais ou aqueles que tiveram um só parceiro sexual e que utilizaram métodos de barreira nas relações sexuais, na categoria "com risco" foram incluídos quem teve um ou mais parceiros sexuais e não se protegeu.

A percepção de risco no trabalho (percepção de susceptibilidade ao realizar funções no Trabalho ou no estudo) foi codificada conferindo pontuação de cinco para sempre e um (1) para nunca.

Os AT-MB analisados neste estudo se referiram àqueles reportados pelos participantes (profissionais e estudantes) e que ocorreram no ano imediatamente anterior, envolvendo exposição a sangue ou fluidos corporais potencialmente contaminados durante o desenvolvimento do trabalho ou estudo ${ }^{19}$.

O questionário foi aplicado individualmente por equipe multidisciplinar previamente treinada (investigadores, estudantes de medicina e psicólogo). As entrevistas foram realizadas no horário e local mais adequado para o participante.

$\mathrm{Na}$ impossibilidade de realizar-se pessoalmente a entrevista, o participante teve a opção de preencher o questionário pela internet em um aplicativo pago da "World Wide Web" para manter a privacidade.

O banco de dados foi digitado utilizando a plataforma Encuestafacil.com, que gera um arquivo padrão 'Excel' que foi posteriormente transferido e analisado no programa SPSS versão 18.0. Para as variáveis categóricas obtivemos as percentagens e para as numéricas calculamos as médias \pm desvios padrão (DP), valores mínimos e máximos e os percentis 25\%, 50\% (mediana) e $75 \%$.

Realizamos a comparação entre os grupos de variáveis categóricas com o teste de qui-quadrado $\left(\chi^{2}\right)$, comparamos médias com o teste de $t$ de Student para amostras independentes, e utilizamos o teste de Kruskal Wallis (KW) para a comparação de medianas.

Recodificamos as escalas de conhecimento e adesão criando novas variáveis, realizando posteriormente os testes de Kolmogorov-Smirnov (K-S) e Shapiro-Wilk (S-W) para determinar o tipo de distribuição das escalas. No caso de a escala ter distribuição normal realizamos a comparação das medias utilizando o teste de Anova para amostras independentes (Brasil-Colômbia), e no caso contrário realizamos o teste de Kruskal -Wallis para comparação de medianas.

Realizamos a Regressão de Poisson-Tweedie ${ }^{20}$ para avaliar a relação das escalas de conhecimen- 
to e de adesão às PPs e às variáveis independentes. Partimos do modelo completo para construir modelos multivariados, empregando todas as variáveis independentes selecionadas para a análise e retirando-as sucessivamente quando não atingissem significância estatística ( $\mathrm{p}>0,05)$. Permaneceram no modelo somente as variáveis estatisticamente significativas com o Intervalo de Confiança de $95 \%$ e o valor de $\mathrm{p}<0,05$.

Calculamos a taxa de incidência para os 12 meses de seguimento em cada país, com base nas somas retrospectivas de meses/pessoa até à data do estudo, estimando coeficientes de incidência para cada país e grupo para poder comparar com a literatura.

O estudo cumpriu com as normas éticas e foi aprovado no Brasil e na Colômbia. Todos os participantes assinaram o Termo de Consentimento Livre e Esclarecido.

\section{Resultados}

\section{Características demográficas}

Avaliamos 965 indivíduos, dos quais 348 eram estudantes (214 no Brasil e 134 na Colômbia) e 617 profissionais (310 no Brasil e 307 na Colômbia), com média (DP) de idade de 33,04 $\pm 10,81$ anos, variando entre 19 a 72 anos; $73,3 \%$ eram do sexo feminino; $71,3 \%$ de cor branca, $58,7 \%$ solteiros, 65,6\% sem Filhos e 42,1\% com renda familiar mensal (RFM) entre seis e 20 salários mínimos mensais (SMM) para o 2014 (Tabela 1).

Ao comparar os dois grupos (estudantes e profissionais) encontramos diferença nas variáveis: idade, cor da pele autorreferida, estado civil, filhos e RFM.

\section{Escalas de Conhecimento, Adesão e Percepção de Risco}

\section{Conhecimento}

A média (DP) de conhecimento nos dois países foi $10,98( \pm 1,34)$ pontos (Mínimo de $0 \mathrm{e}$ Máximo de 12), mediana de 11 pontos $(10,12)$ comparada à média mínima esperada de 9 pontos. O $\alpha$-Cr da escala foi de 0,625 e não apresentou distribuição normal (Tweedie) (teste K-S p < 0,001). Quando consideramos 9 pontos ou mais como ponto de corte para ter bom conhecimento ${ }^{21}$ encontramos 95,1\% (915) dos participantes nesta categoria (Tabela 1 ).

\section{Adesão às Precauções Padrão (PPs)}

A Adesão às precauções padrão teve média (DS) de 30,74 $( \pm 4,51)$ (Mínimo $=0$ e Máximo = 36; Mínima esperada $=27,75)$, e mediana de 31 pontos (percentil $25=28$ e percentil $75=34$ ). O coeficiente $\alpha$-Cr da escala foi de 0,745 quando eliminamos as perguntas sobre reencape de perfurocortantes e vacinação contra a Hepatite B. A escala apresentou distribuição de Tweedie (teste $\mathrm{K}-\mathrm{S} \mathrm{p}>0,001)$.

Avaliamos que $83,1 \%$ dos participantes tiveram boa adesão ( $\geq 27,75$ pontos). Quando avaliadas as diretrizes separadamente, encontramos que $56,3 \%$ sempre lavaram as mãos antes e depois de examinar o paciente, $51,0 \%$ antes e depois de usar luvas e $94,6 \%$ ao entrar em contato com fluidos corporais. Sobre o uso de Elementos de Proteção Pessoal (EPPs) verificamos que $86,0 \%$ usam sempre luvas, 36,9\% usam óculos, $56,0 \%$ usam máscara e 58,7\% usam jaleco ou vestuário cirúrgico.

O descarte de perfurocortante é realizado sempre em coletores por $72,2 \%$ dos participantes, e $2,4 \%$ nunca realizam o reencape, mas $93,5 \%$ o fazem sempre, o que é mais prevalente entre os profissionais $(60,0 \%)$, em especial os enfermeiros quando comparados com os estudantes $(33,5 \%)$.

Dos 957 participantes que responderam à pergunta, $835(87,27 \%)$ reportaram ter tomado pelo menos uma dose da vacina contra o vírus da Hepatite B, e dentre eles 184 (22,03\%) relataram ter tomado três doses; $63(7,54 \%)$ relataram ter tomado um reforço e 33 (3,95\%) repetiram as três doses. Não houve diferença vacinal entre os grupos. Os profissionais da saúde tiveram prevalência de $89,3 \%$ em comparação a $68,7 \%$ dos estudantes.

A confirmação da imunidade (AntiHBs) por exame sorológico foi mencionada por 665 $(69,6 \%)$ participantes e 499 (75,3\%) indicaram saber que estavam imunizados. Encontramos diferença ( $p<0,001)$ entre os grupos (profissionais e estudantes). Os profissionais fizeram o teste sorológico em maior percentagem (74,0\% $>26,0 \%$ ) e os que relataram acidentes também testaram a imunidade (AntiHBs) em maior proporção $(92,3 \%>80,4 \%)$.

\section{Percepção de risco}

Encontramos média de $3,15( \pm 1,10)$ pontos (Mínimo de 1 e Máximo de 5), mediana de 3 pontos, para média mínima esperada de 3,75 pontos. A escala apresentou distribuição normal (teste K-S p > 0,428). Não encontramos diferen- 
Tabela 1. Comparação das características sociodemográficas e comportamentais entre os participantes, Brasil Colômbia, 2015.

\begin{tabular}{|c|c|c|c|c|}
\hline Variáveis & $\mathbf{N}$ & $\begin{array}{c}\text { Brasil } \\
\mathrm{n}=524(\%)\end{array}$ & $\begin{array}{c}\text { Colômbia } \\
\mathrm{n}=441(\%)\end{array}$ & $\mathbf{p}$ \\
\hline Gênero & & & & 0,671 \\
\hline Feminino & 707 & $381(53,9)$ & $326(46,1)$ & \\
\hline Masculino & 258 & $143(55,4)$ & $115(44,6)$ & \\
\hline Idade & & & & $0,354^{*}$ \\
\hline Mediana (percentis 25,75 ) & & $30(23,39)$ & $30(25,41)$ & \\
\hline Média \pm DP & 965 & $32,75 \pm 10,81$ & $33,39 \pm 10,81$ & \\
\hline Mínimo & & 20 & 19 & \\
\hline Máximo & & 64 & 72 & \\
\hline Cor da Pele & & & & 0,001 \\
\hline Branca & 688 & $417(60,6)$ & $271(39,4)$ & \\
\hline Negro-Pardo & 118 & $76(64,4)$ & $42(35,6)$ & \\
\hline Amarela & 25 & $23(92,0)$ & $2(8,0)$ & \\
\hline Indígena - Mestiço & 92 & $1(1,1)$ & $91(98,9)$ & \\
\hline Não Sabe & 42 & $7(16,7)$ & $35(83,3)$ & \\
\hline Estado Civil - Risco & & & & 0,001 \\
\hline Solteiro/Viúvo/Separado & 650 & $328(50,5)$ & $322(49,5)$ & \\
\hline Casado/União Estável & 315 & $196(62,2)$ & $119(37,8)$ & \\
\hline Número de Filhos & & & & 0,004 \\
\hline Sem Filhos & 631 & $364(57,7)$ & $267(42,3)$ & \\
\hline Com Filhos & 333 & $160(48,0)$ & $173(52,0)$ & \\
\hline $\begin{array}{l}\text { Renda Familiar } \\
\text { (SMM Salário Mínimo Mensal) }\end{array}$ & & & & 0,001 \\
\hline$<1 \mathrm{SMM}$ & 24 & $2(8,3)$ & $22(91,7)$ & \\
\hline 1 a 5 SMM & 399 & $135(33,8)$ & $264(66,2)$ & \\
\hline 6 a 20 SMM & 403 & $295(73,2)$ & $108(26,8)$ & \\
\hline 21 ou mais SMM & 90 & $65(72,2)$ & $25(27,8)$ & \\
\hline Não Sabe & 49 & $27(55,1)$ & $22(44,9)$ & \\
\hline Comportamento de Risco Sexual & & & & 0,561 \\
\hline Sim & 515 & $285(55,3)$ & $230(44,7)$ & \\
\hline Não & 445 & $237(53,3)$ & $208(46,7)$ & \\
\hline Conhecimento & & & & 0,001 \\
\hline Bom & 918 & $513(55,9)$ & $405(44,1)$ & \\
\hline Mal & 47 & $11(23,4)$ & $36(76,6)$ & \\
\hline Adesão às PPs & & & & 0,001 \\
\hline Bom & 745 & $439(58,9)$ & $306(41,1)$ & \\
\hline Mal & 197 & $73(37,1)$ & $124(62,9)$ & \\
\hline Percepção de Risco & & & & 0,986 \\
\hline Alta & 249 & $135(54,2)$ & $114(45,8)$ & \\
\hline Baixa & 689 & $374(54,3)$ & $315(45,7)$ & \\
\hline Treinamento & & & & 0,486 \\
\hline Sim & 133 & $76(57,1)$ & $57(42,9)$ & \\
\hline Não & 809 & $436(53,9)$ & $373(46,1)$ & \\
\hline Acidente & & & & 0,686 \\
\hline Sim & 103 & $54(52,4)$ & $49(47,6)$ & \\
\hline Não & 862 & $471(54,6)$ & $392(45,4)$ & \\
\hline
\end{tabular}

Nota: $\mathrm{p}\left(\right.$ Teste $\mathrm{X}^{2}$ quadrado e Exato de Fisher para as variáveis categóricas; ${ }^{*}$ ANOVA para comparação de medias em amostras independentes).

ça entre os países, ainda que entre os grupos ( $\mathrm{p}$ $<0,002)$, os profissionais tinham percepção de risco $(3,25 \pm 1,13)$ maior que os estudantes $(2,97$ $\pm 1,04)$.
Fatores associados ao conhecimento e adesão às PPs

Entre os fatores associados ao nível de conhecimento encontramos, após ajuste dos modelos, 
que são importantes país de origem, profissão, ter o esquema completo de vacinação contra o VHV, realização do AntiHBs, ter sofrido acidente e ter filhos (Tabela 2).

A Tabela 3 apresenta os resultados da Regressão de Poisson-Tweedie realizada para avaliar os fatores associados à adesão às PPs. O melhor modelo ajustado associou país, grupo (estudante), subgrupo (estudantes do Brasil), estado civil (risco), acidente e percepção de risco às PPs.

Os fatores associados ao nível de adesão entre os profissionais da saúde foram: idade $(\mathrm{p}<0,031$ : IC 95\% 0,000-0,003), tipo de área ( $\mathrm{p}<0,023$ : IC 95\% 0,004-0,053), País ( $<$ 0,001: IC 95\% $0,033-0,084)$, vacina contra HBV ( $p<0,001$ : IC 95\% 0,027-0,115), acidente ( $\mathrm{p}<0,018$ : IC 95\%
$-0,093$ a $-0,021)$, receber treinamento $(\mathrm{p}<0,033$ : IC 95\% 0,015-0,074), e percepção de risco ( $\mathrm{p}<$ 0,032: IC 95\% 0,001-0,023).

\section{Acidentes de trabalho}

Entre os participantes avaliados, 103 (10,7\%) referiram ter sofrido acidente com material biológico nos 12 meses imediatamente anteriores sem diferença entre os países (Tabela 4). A taxa de incidência de acidentes foi de 6,05 por 100 pessoas/ano nos dois países; no Brasil foi de 7,5 casos por pessoa/ano e na Colômbia de 3,5 casos por pessoas/ano.

Ao avaliar os fatores associados a sofrer AT -MB encontramos que o comportamento Sexual de Risco $(\mathrm{p}<0,050)$, percepção de risco no

Tabela 2. Modelo de Regressão Poisson-Tweedie para determinar os fatores associados ao nível de conhecimento entre profissionais e estudantes da saúde, Brasil - Colômbia, 2015.

\begin{tabular}{|c|c|c|c|c|c|c|}
\hline \multirow{2}{*}{ Variáveis } & \multicolumn{3}{|c|}{ Análise Simples } & \multicolumn{3}{|c|}{ Análise Multivariada } \\
\hline & B & IC95\% & p-valor & Bajud * & IC95\% & p-valor \\
\hline Idade em anos & 0,001 & $-0,001-0,001$ & 0,538 & & & \\
\hline Sexo & & $-0,007$ a $-0,002$ & 0,091 & & & \\
\hline Feminino & 0 & & & & & \\
\hline Masculino & 0,013 & & & & & \\
\hline Estado Civil - Risco & & $0,004-0,004$ & 0,004 & & & \\
\hline Solteiro/Viúvo/Separado & 0 & & & & & \\
\hline Casado/União Estável & 0,019 & & & & & \\
\hline Filhos & & $0,007-0,060$ & 0,012 & 0,029 & $0,006-0,05$ & 0,013 \\
\hline $\mathrm{Si}$ & 0 & & & & & \\
\hline No & 0,031 & & & & & \\
\hline País & & 0,07 a 0,05 & 0,001 & 0,069 & 0,05 a 0,08 & 0,001 \\
\hline Brasil & 0,064 & & & & & \\
\hline Colômbia & 0 & & & & & \\
\hline Grupo & & $-0,004-0,029$ & 0,139 & & & \\
\hline Profissionais & 0 & & & & & \\
\hline Estudantes & 0,012 & & & & & \\
\hline Risco Sexual & & $-0,016-0,016$ & 0,985 & & & \\
\hline Sim & 0 & & & & & \\
\hline Não & 0,001 & & & & & \\
\hline Vacina de Hepatite B & & $-0,073-0,015$ & 0,003 & $-0,042$ & $-0,067 \mathrm{a}-0,018$ & 0,001 \\
\hline Sim & 0 & & & & & \\
\hline Não & $-0,044$ & & & & & \\
\hline Realização AntiHBs & & $-0,045-0,006$ & 0,010 & $-0,027$ & $-0,046 \mathrm{a}-0,009$ & 0,004 \\
\hline Sim & 0 & & & & & \\
\hline Não & $-0,025$ & & & & & \\
\hline Acidente com MB & & $-0,042$ a $-0,005$ & 0,014 & $-0,022$ & $-0,040 a-0,004$ & 0,017 \\
\hline Sim & 0 & & & & & \\
\hline Não & $-0,023$ & & & & & \\
\hline Treinamento & & $-0,004$ a 0,026 & 0,167 & & & \\
\hline Sim & 0 & & & & & \\
\hline Não & 0,011 & & & & & \\
\hline Escala de adesão & 0,001 & $-0,002$ a 0,002 & 0,758 & & & \\
\hline Escala de Percepção Risco & 0,003 & 0,0042 a 0,009 & 0,127 & & & \\
\hline
\end{tabular}


Tabela 3. Modelo de Regressão Poisson-Tweedie para determinar os fatores associados ao nível de adesão às PPs entre profissionais e estudantes da saúde, Brasil - Colômbia, 2015.

\begin{tabular}{|c|c|c|c|c|c|c|}
\hline \multirow{2}{*}{ Variáveis } & \multicolumn{3}{|c|}{ Análise Univariada } & \multicolumn{3}{|c|}{ Análise Multivariada } \\
\hline & B & IC95\% & p-valor & Bajud * & IC95\% & p-valor \\
\hline Idade em anos & 0,00 & $-0,01-0,01$ & 0,783 & & & \\
\hline \multicolumn{7}{|l|}{ Sexo } \\
\hline \multicolumn{7}{|l|}{ Feminino } \\
\hline Masculino & 0,008 & $-0,015-0,031$ & 0,483 & & & \\
\hline \multicolumn{7}{|l|}{ Estado Civil - Risco } \\
\hline Solteiro/Viúvo/Separado & $-0,021$ & $-0,004-0,002$ & 0,077 & $-0,031$ & $-0,058 \mathrm{a}-0,003$ & 0,027 \\
\hline Casado/União Estável & 0 & & & & & \\
\hline \multicolumn{7}{|l|}{ Filhos } \\
\hline $\operatorname{Sim}$ & 0 & & & & & \\
\hline Não & $-0,003$ & $-0,024-0,018$ & 0,819 & & & \\
\hline \multicolumn{7}{|l|}{ País } \\
\hline Brasil & 0,57 & 0,029 a 0,086 & 0,001 & 0,067 & $0,048-0,086$ & 0,001 \\
\hline Colômbia & 0 & & & 0 & & \\
\hline \multicolumn{7}{|l|}{ Grupo } \\
\hline Profissionais & 0 & & & 0 & & \\
\hline Estudantes & 0,037 & 0,019-0,056 & 0,001 & 0,058 & $0,048-0,086$ & 0,001 \\
\hline \multicolumn{7}{|l|}{ Subgrupo } \\
\hline Profissionais Brasil & 0 & & & 0 & & \\
\hline Profissionais Colômbia & $-0,064$ & $-0,09$ a $-0,038$ & 0,001 & 0 & & \\
\hline Estudantes Brasil & 0,033 & $0,011-0,054$ & 0,003 & 0,057 & $0,027-0,086$ & 0,001 \\
\hline Estudantes Colômbia & $-0,041$ & $-0,07$ a $-0,026$ & 0,001 & 0,038 & $0,055-0,072$ & 0,025 \\
\hline \multicolumn{7}{|l|}{ Risco Sexual } \\
\hline Sim & 0 & & & & & \\
\hline Não & $-0,007$ & $-0,025-0,014$ & 0,504 & & & \\
\hline \multicolumn{7}{|l|}{ Vacina de Hepatite B } \\
\hline $\operatorname{Sim}$ & 0,064 & $0,033-0,095$ & 0,045 & & & \\
\hline Não & 0 & & & & & \\
\hline \multicolumn{7}{|l|}{ Realização AntiHBs } \\
\hline Sim & 0 & & & & & \\
\hline Não & $-0,015$ & $-0,033-0,095$ & 0,168 & & & \\
\hline \multicolumn{7}{|l|}{ Acidente com MB } \\
\hline $\operatorname{Sim}$ & $-0,063$ & $-0,099$ a $-0,026$ & 0,001 & $-0,057$ & $-0,093$ a- 0,021 & 0,001 \\
\hline Não & 0 & & & 0 & & \\
\hline \multicolumn{7}{|l|}{ Treinamento } \\
\hline $\operatorname{Sim}$ & 0 & & & & & \\
\hline Não & 0,019 & $-0,009$ a 0,048 & 0,183 & & & \\
\hline Escala de conhecimento & 0,009 & $-0,001$ a 0,018 & 0,070 & & & \\
\hline Escala de Percepção Risco & 0,008 & $-0,002$ a 0,019 & 0,215 & 0,012 & $0,001-0,023$ & 0,036 \\
\hline
\end{tabular}

trabalho ( $\mathrm{p}<0,023)$, realização do AntiHBs ( $\mathrm{p}$ $<0,002)$, conhecimento $(\mathrm{p}<0,018)$ e adesão às PPs $(\mathrm{p}<0,039)$ estão relacionados com o evento. Quando analisamos os profissionais da saúde (médicos e enfermeiros) separadamente dos estudantes, os AT-MB estiveram associados às ho- ras trabalhadas ( $\mathrm{p}<0,009)$, ao tipo de especialização (Cirurgia e Clínica) ( $\mathrm{p}<0,005)$, e à adesão às PPs $(\mathrm{p}<0,001)$.

\section{Comportamento sexual de risco}

Encontramos que 75,5\% dos entrevistados apresentaram comportamento sexual de risco, 
Tabela 4. Características dos acidentes com material biológico entre estudantes e profissionais da saúde, Brasil-Colômbia, 2015.

\begin{tabular}{|c|c|}
\hline Variáveis & $\begin{array}{c}\text { Ano Anterior } \\
(965)\end{array}$ \\
\hline \multicolumn{2}{|l|}{ Acidente } \\
\hline Sim & $103(10,7)$ \\
\hline Não & $862(89,3)$ \\
\hline \multicolumn{2}{|l|}{ Horário } \\
\hline Diurno & $43(41,7)$ \\
\hline Tarde & $26(25,2)$ \\
\hline Noturno & $18(17,5)$ \\
\hline Não Sabe/Não Lembra & $16(15,5)$ \\
\hline \multicolumn{2}{|l|}{ Dia da Semana } \\
\hline Segunda & $2(1,9)$ \\
\hline Terça & $4(3,9)$ \\
\hline Quarta & $2(1,9)$ \\
\hline Quinta & $8(7,8)$ \\
\hline Sexta & $9(8,8)$ \\
\hline Sábado o Domingo & $8(7,8)$ \\
\hline Não Sabe/No Lembra & $70(67,9)$ \\
\hline \multicolumn{2}{|l|}{ Dia da Semana } \\
\hline Segunda a Sexta & $25(24,3)$ \\
\hline Sábado o Domingo & $8(7,8)$ \\
\hline Não Sabe/Não Lembra & $70(67,9)$ \\
\hline \multicolumn{2}{|l|}{ Objeto Causa do Acidente } \\
\hline Agulha & $67(65,0)$ \\
\hline Cateter, Gelco e outros & $12(11,7)$ \\
\hline Bisturi & $6(5,8)$ \\
\hline Fio e outros & $7(6,8)$ \\
\hline Sangue e Fluidos & $11(10,7)$ \\
\hline \multicolumn{2}{|l|}{ Parte do Corpo Afetada } \\
\hline Dedos & $70(68,0)$ \\
\hline Mão & $23(22,3)$ \\
\hline Rosto & $7(6,8)$ \\
\hline Braços & $3(2,9)$ \\
\hline \multicolumn{2}{|l|}{ Reportou o acidente } \\
\hline $\operatorname{Sim}$ & $76(73,8)$ \\
\hline Não & $27(26,2)$ \\
\hline \multicolumn{2}{|l|}{ Uso de EPPs } \\
\hline Sim & $72(69,9)$ \\
\hline Não & $31(30,1)$ \\
\hline \multicolumn{2}{|l|}{$\begin{array}{c}\text { Perfuro cortante com } \\
\text { dispositivo de segurança }\end{array}$} \\
\hline Sim & $11(13,9)$ \\
\hline Não & $68(86,1)$ \\
\hline
\end{tabular}

Nota: EPPs Elementos de Proteção Pessoal.

sem diferença entre os países (Tabela 1); encontrando-se associado com estado civil (casado/ união estável vs solteiro/viúvo/separado) $\mathrm{RP}=$ 1,67 (IC95\% 1,34-2,07) e acidente com material biológico RP = 1,63 (IC95\% 1,03-2,85).

O número de parceiros sexuais no último ano foi em média de 1,29 ( $\pm 1,04)$ variando de 0 a 6 , com diferença segundo o grupo e gênero. $\mathrm{O}$ uso de proteção nas relações sexuais não foi utilizado por $62,7 \%$, e dos $37,6 \%$ que referiram ter-se protegido, $35 \%$ utilizaram preservativo sem diferença entre os países.

\section{Treinamento}

Encontramos que $34,9 \%$ dos participantes referiram a não existência de treinamento próprio, existindo diferença entre os países $(\mathrm{p}<$ 0,003 ), este fato é referido com maior frequência no Brasil (21,4\%) (Tabela 1).

Em relação à frequência 38,9\% (369) relataram a irregularidade do treinamento e $28,3 \%$ (269) não sabiam quando foi realizado. Encontramos diferença entre os países ( $p<0,001)$, pois no Brasil o desconhecimento é maior.

\section{Aspetos que fazem fácil ou difícil a adesão às PPs}

A adesão às PPs melhora com a capacitação, o treinamento continuo e regular, e informações em forma de avisos e pôsteres, especialmente. Entre os aspectos que dificultam a adesão, encontramos a falta de disponibilidade do material $(51,4 \%)$, a sobrecarga de trabalho $(18,3 \%)$, a pressa $(16,8 \%)$ e o difícil acesso aos EPPs $(13,5 \%)$.

\section{Discussão}

As características demográficas sexo e idade entre os estudantes são semelhantes a estudos no Canadá $^{22}$ e nos Estados Unidos ${ }^{23}$; e entre os profissionais na Colômbia ${ }^{24}$ e Irã ${ }^{25}$.

O maior número de mulheres no presente estudo $(73,3 \%)$ se deve à predominância do sexo feminino entre os profissionais de enfermagem e à relação mulher:homem (2:1) entre todos os profissionais.

Considerando os resultados do presente estudo podemos afirmar que os Acidentes de Trabalho com Material Biológico (AT-MB) continuam sendo um problema de Saúde Pública - Coletiva e mundial, apesar das múltiplas intervenções realizadas ao longo do tempo; desde as recomendações sobre lavar as mãos do Dr. Semmelweis até as diretrizes do $\mathrm{CDC}^{9}$ e da Administração de Segurança e Saúde do trabalho (OSHA), fortalecidas no Ato Legislativo de $2000^{26}$, continuando em 2005 no Brasil com a publicação da Norma Regulamentadora NR-32 até, mais recentemente, na União Europeia com o Acordo-Quadro 2010/32/ $\mathrm{UE}^{27}$, que tem como foco a implantação dos dispositivos de segurança nos perfurocortantes. 
Encontramos coeficientes ou proporções de incidência de 10,3\% no ano anterior à constituição da coorte e de 5,5\% no ano de seguimento no Brasil que podem ser comparados com dados nacionais do período posterior à publicação da NR-32 em 2005, que reportam 185.910 AT-MB no período de 2007 a 2013, com coeficientes de incidência que passaram de 1,47\% em 2007 para $3,70 \%$ em 2013. Estes números podem expressar tanto o aumento da notificação ou a redução da subnotificação, assim como a tendência a um aumento real das proporções de incidência nos ambientes de trabalho da Saúde no Brasil durante o período $^{28}$.

Os coeficientes de Prevalência de Relato de Incidentes de AT-MB no ano anterior ao estudo (5,5 AT-MB/100 pessoas/ano no estudo binacional) foram maiores que os reportados em estudos internacionais ${ }^{29,30}$ e nacionais ${ }^{31}$.

Os resultados sobre as características do acidente vão ao encontro das observações em estudos no Brasil $^{32}$, na Colômbia ${ }^{33}$ e em outros Países $^{34}$. Quando avaliamos os fatores associados aos AT-MB, encontramos dados similares aos relatados tanto no Brasil como em outros países. Entre os fatores mais relevantes encontramos a área de atuação (cirúrgica e clínica) ${ }^{33,35}$, profissão (médica) $)^{35,36}$, horas trabalhadas por semana $(>42$ horas $)^{35,37}$, conhecimento ${ }^{38}$, adesão às precauções padrão ${ }^{36}$ e percepção de risco ${ }^{29}$.

O nível de conhecimento entre os participantes do estudo foi alto quando comparamos com estudo que utilizou a mesma escala (2,7 e 5,7 pontos) no Paquistão ${ }^{38}$; encontrando-se melhor conhecimento entre os brasileiros, os médicos e os quem têm filhos.

Também os que sofreram AT-MB, apresentaram níveis de conhecimento maior; explicável pelo fato de que ao sofrer um acidente nos questionamos sobre estes temas e procuremos informação.

Percebemos adicionalmente que os participantes que têm altos níveis de conhecimento realizam com maior prevalência o teste de Anti-HBs e completam o esquema de vacinação contra o vírus da Hepatites B, sendo um reflexo tanto da exposição acidental como da procura de informação.

Neste estudo encontramos média de adesão às $\mathrm{PPs}$ de $30,74 \pm 4,51$ pontos, para um esperado de 27,75; o que levou a concluir que o nível de adesão era bom. Ao avaliar separadamente cada uma das diretrizes notamos, entretanto, que a adesão ao uso de óculos (2,6 pontos) e ao não reencape de perfurocortantes (2,44 pontos) eram ainda baixas, similares às do estudo realizado com médicos em Hospital universitario ${ }^{35}$, sendo uma atitude que expõe o profissional ou o estudante ao risco de adquirir doenças ao contato com fluidos corporais.

Sabemos que a adesão às medidas de proteção pode ser compreendida como um conjunto de categorias de resposta cuja característica comum é o seguimento de orientações fornecidas ou recomendadas por outros ${ }^{39}$, dependendo de muitos fatores, mas especialmente da percepção do risco como escrito no presente estudo e que é equivalente ao estudo citado que utilizou escala similar ${ }^{38}$.

Entre outros fatores associados à baixa adesão às PPs encontramos o estado civil. Esse dado chama a atenção porque ser solteiro, viúvo ou separado é fator de risco tanto para comportamentos de risco sexual como para atitudes de risco ocupacional. Estas descobertas são similares às encontradas entre médicos de hospital universitário em Belo Horizonte ${ }^{35}$.

Destacamos que indivíduos que não tem responsabilidades familiares diminuem seus níveis de proteção contra todo tipo de risco. Estudos realizados na América Latina informam que os solteiros se expõem mais aos riscos, tanto sexuais $^{40}$ como ocupacionais ${ }^{41}$ e de comportamentos em geral, o que inclui dirigir em altas velocidades sem cinto de segurança.

Dentre outros fatores associados à baixa adesão às precauções (PPs) encontramos sofrer AT-MB, como descrito em estudo realizado no Paquistão ${ }^{36}$ que relata que uma boa adesão à PPs se reflete em menores prevalências de acidentes (RP 0,94).

Inversamente ao esperado, quem sofreu AT -MB, percebia mais o risco de adquirir doenças pelo trabalho desenvolvido, possivelmente como resposta à exposição. Nossos dados parecem confirmar isso já que os participantes com maior prevalência de acidentes foram os profissionais $(63,7 \%)$, e são eles que têm níveis de percepção do risco também maiores. Isto concorda com o descrito por Corrêa-Filho ${ }^{14}$ e Cordeiro ${ }^{12}$, que afirmam que os trabalhadores que sofrem lesões no trabalho têm percepção diferenciada em relação aos fatores de risco imediato na historia de seus acidentes.

A prevalência de esquemas completos de vacinação contra a Hepatite B foi de 81,9\%, similar aos resultados de estudos brasileiros $(73,5 \%$ y $97,7 \%)^{35}$.

Em relação à realização do teste de anti-HBs, encontramos semelhanças com pesquisa condu- 
zida no Irã, onde $60 \%$ dos profissionais testaram sua imumidade ${ }^{42}$; e entre os participantes que fizeram o teste, $69,1 \%$ reportaram possuir imunidade, dado igual ao obtido em Unidade Básica de Saúde no Brasil ${ }^{43}$.

É importante ressaltar que entre os acidentados existem as maiores prevalências tanto em esquemas de vacinação como na realização do teste AntiHBs; fato secundário ao ingresso deste grupo nos protocolos de seguimento.

Conforme nossa descrição e os resultados em relação ao país de origem dos participantes, que confirmam a existência de níveis de conhecimento e adesão superiores no Brasil em relação à Colômbia, podemos concluir que Normas como a Brasileira, publicada com o propósito de proteger os trabalhadores da saúde de acidentes e doenças especificas da profissão, fortalecem os conhecimentos sobre os meios de transmissão de múltiplos micro-organismos e aumentam a adesão às precauções padrão. Entretanto, estes não são meios totalmente eficazes para eliminar a ocorrência dos acidentes com material biológico.

Mudanças nos aspectos organizacionais que não foram incluídos na norma, especialmente referentes à sobrecarga de trabalho e número de horas trabalhadas por semana, contribuiriam mais eficazmente neste aspecto.

Finalmente, lembramos que cada uma das diretrizes sobre risco biológico na norma NR-32 tem o objetivo de garantir as boas praticas no uso e descarte de perfurocortantes e assim diminuir a incidência de acidentes; especialmente com a implantação dos dispositivos de segurança. Estudos realizados nos EUA evidenciaram que a publicação do Ato legislativo de $2000^{26}$ contribuiu para a redução de incidentes com materiais cortantes e perfurocortantes $^{44}$.

Porém, para poder conseguir melhores atitudes de proteção que contribuam para verdadeiras mudanças de comportamento, é necessário treinamento adicional contínuo, como descrito na norma NR-32. Assim, propomos o fortalecimento da percepção de risco entre os trabalhadores e os estudantes da área da Saúde, por meio de treinamentos e avaliações periódicas, em todos os níveis da formação e no trabalho.

Entre os principais fatores descritos pelos participantes como facilitadores da adesão encontramos: treinamento, acesso às informações por cartazes e avisos nos locais de trabalho, uso de equipamentos de proteção individual (EPIs) e disponibilidade dos mesmos, semelhante ao exposto pelos participantes de outros estudos brasileiros ${ }^{45}$.
Estudo brasileiro descreveu entre os aspectos que dificultam a adesão às PPs: a sobrecarga de trabalho, a falta de treinamento específico, a dificuldade na adaptação aos EPIs, a falta de tempo e a falta de EPIs, de incentivos e hábitos. Estes aspectos formam muito similares aos expressados pelos participantes do presente estudo ${ }^{45}$.

Entre outros fatores reportados pelos profissionais e estudantes que dificultam a adesão às PPs encontramos a pressa e a urgência, conforme corroborado por estudo anterior que acredita que a rotina do serviço pesada e debilitante minimize o sentimento de medo gerado pela possibilidade de sofrer acidentes e ter consequências graves. Entretanto, esse sentimento de pânico não impede o risco de acidentar-se e secundariamente contaminar-se, o que torna necessário criar situações de treinamento continuo ${ }^{46}$.

Esclarecemos que os sistemas de Saúde no Brasil e na Colômbia na atualidade são diferentes $^{47}$; mas, o Hospital Militar Central onde se desenvolveu o estudo na Colômbia ainda é uma fenda da presença do Estado no sistema, existindo regimes denominados especiais, nos quais grupos específicos (militares, trabalhadores públicos e petroleiros) têm assistência integral e acesso universal. No Brasil se mantém o principio da solidariedade e o conceito de cidadania, estruturando-se modelos como o Sistema Único de Saúde (SUS), que garante o acesso umiversal ${ }^{50}$, sendo similar à fissura encontrada no Hospital colombiano que fez parte deste estudo. Além disso, é possível comparar os participantes dos países inclusos no estudo tanto pelas similitudes no processo de trabalho no sistema de saúde como do modelo biomédico de aprendizagem aos quais os participantes estão expostos.

\section{Conclusões}

A proporção ou o coeficiente de incidência prévia de AT-MB, entre profissionais e estudantes da saúde foi alta, sendo maiores no Brasil que na Colômbia. Os participantes do Brasil, estudantes, entre os profissionais os médicos que atuam nas áreas cirúrgicas ou unidades de cuidados intensivos e que trabalham por mais de 42 horas por semana tiveram mais AT-MB.

O nível de conhecimento foi bom, sendo melhor entre os participantes do Brasil, possivelmente como resultado da implantação da NR-32, e esteve associado ao País de origem, a ter filhos (estado civil casado), cumprir o esquema completo de vacinação, realizar o teste do AntiHBs 
e sofrer AT-MB, e não foi um bom preditor da adesão às PPs.

O nível de adesão às PPs foi bom, mas quando avaliadas as diretrizes separadamente, a adesão ao uso de óculos e o não reencape de agulhas foi baixo. Fatores como o país de origem (Brasil), grupo (estudante brasileiro), estado civil (casado/união estável), não sofrer acidente e a percepção de risco estão associados a melhores níveis de adesão às PPs.

\section{Colaboradores}

EIG La-Rotta foi a idealizadora do projeto e com FH Aoki e HR Correa-Filho desenharam o estudo. CM Pertuz, DD Trevisan, AR Camisão e CS Garcia coletaram dados. CM Pertuz, CS Garcia e IOC Miquilin auxiliaram nas análises dos dados e na escrita do texto em conjunto com EIG La-Rotta. EIG La-Rotta, HR Correa-Filho e FH Aoki escreveram o manuscrito. Todos os autores leram e aprovaram o manuscrito.

\section{Agradecimentos}

Aos participantes. Às Diretivas dos Hospitais de Clínicas da Unicamp no Brasil e Hospital Militar Central na Colômbia. À FAEPEX (Brasil) e à Universidade El Bosque (Colômbia) pela concessão de apoio econômico. "O presente trabalho foi realizado com apoio do Programa Estudiantes Convênio de Pós-Graduação - PEC-PG, de CA$\mathrm{PES} / \mathrm{CNPq}$ - Brasil”. Os autores agradecem ao Espaço da Escrita - Coordenadoria Geral da Universidade - UNICAMP - pelo serviço de tradução ao inglês do texto. 


\section{Referências}

1. Prüss Üstün A, Rapiti E, Hutin Y. Estimation of the global burden of disease attributable to contaminated sharps injuries among health care workers. American $J$ Industrial Medicine 2005; 48(6):482-490.

2. Vargas-Silva DC, Prieto-Moreno JA. Caracterización de las variables de los accidentes de trabajo biológicos en el personal de servicios generales que labora en diversas instituciones hospitalarias, afiliadas a una Administradora de Riesgos Laborales [tesis]. Bogotá: Univeridad Javeriana; 2012.

3. Correa-Alvarado D, Parada-Rincón D. Accidentes con riesgo biológico en una población afiliada a una $A R L$ [tese]. Bogotá: Univeridad El Rosario; 2014.

4. Brasil. Ministério da Economia (ME). Anuário Estatístico da Previdência Social. Brasilia: ME; 2015. Vol. 24

5. Departamento Intersindical de Estatística e Estudos Socioeconômicos (DIEESE). Anuário da saúde do trabalhador. São Paulo: DIEESE; 2016.

6. Oliveira BR, Murofuse N. Occupational accidents and occupational disease: study of the hospital workers' knowledge about health risks of their work. Rev Lat Am Enfermagem 2001; 9(1):109-115.

7. Rapparini C. Riscos Biológicos e Segurança dos Profissionais da Saúde. SBI-Infectologia Hoje 2006; 1(2):88.

8. CDC. Guidelines for the Management of Occupational Exposures to HBV, HCV and HIV and Recommendations for Postexposure Prophylaxis. MMWR. 2001; 50(11):3-7.

9. Roberts C. Universal precautions: improving the knowledge of trained nurses. Br J Nurs 2000; 9(1):4347.

10. Ferguson J. Preventing healthcare-associated infection: risks, healthcare systems and behaviour. Intern Med J 2009; 39(9):574-581.

11. Saleem T, Khalid U, Ishaque S, Zafar A. Knowledge, attitudes and practices of medical students regarding needle stick injuries. The J the Pakistan Medical Association 2010; 60(2):151.

12. Cordeiro R. Suggestion of an inverse relationship between perception of occupational risks and work-related injuries. Cad Saude Publica 2002; 18(1):45-54.

13. Dela-Coleta J, Silva L, Freitas L, Peters L. As causas dos acidentes de trabalho para operários acidentados e näo acidentados, chefias e supervisores de segurança. Revista Brasileira de Saúde Ocupacional 1989; 14(53):42-51.

14. Corrêa-Filho H. Percepção de Risco na ocupação precedendo lesões do trabalho: Um estudo no municipio de Campinas, São Paulo, 1992 - 1993 [tese]. São Paulo: Universidade de São Paulo; 1994.

15. Breilh J, Granda E, Campaña A, Yépez J, Páez R, Costales P. Deterioro de la vida: un instrumento para análisis de prioridades regionales en lo social y la salud. Quito: CEAS; 1990.

16. Toledo A. Conhecimentos, atitudes e comportamentos frente ao risco ocupacional de exposição ao HIV entre estudantes de Medician da Faculdade de Medicina da Universidade de Minas Gerais. Rev Soc Bras Med Trop 1999; 32(5):509-515.
17. Lopes $\mathrm{A}$, Oliveira $\mathrm{A}$, Silva J, Paiva $\mathrm{M}$. Adesão às precauções padrão pela equipe do atendimento pré-hospitalar móvel de Belo Horizonte, Minas Gerais, Brasil. Cad Saude Publica 2008; 24(6):1387-1396.

18. Gomez-La-Rotta E, Aoki F, Stephan C, Luz V, Pereira F, Ortega-Mora G, Correa-Filho H. Conhecimento e adesão às precauções padrão: Estudantes diante dos riscos biológicos no Brasil e na Colômbia. Revista de Salud Pública. 2015; 17(5):429-442.

19. Centro de Referência em Saúde do Trabalhador (CEREST). Estatisticas dos agravos relacionados ao trabalho, Acidentes com Material Biológico. Natal: CEREST; 2010.

20. Carrasco-Peña M, González J. Modelización de conteos mediante la distribución Poisson-Tweedie (PT):aplicación en datos de ultrasecuenciación [TCC]. Barcelona: Universidad Autonoma; 2013.

21. Sax H, Perneger T, Hugonnet S, Herrault P, Chraiti MN, Pittet D. Knowledge of standard and isolation precautions in a large teaching hospital. Infect Control Hosp Epidemiol. 2005; 26(3):298-304.

22. Ouyang B, Li LD, Mount J, Jamal A, Berry L, Simone C, Law M, Tai R. Incidence and Characteristics of Needlestick Injuries among Medical Trainees at a Community Teaching Hospital: A Cross-Sectional Study. J Occupational Health 2017; 59(1):63-73.

23. Choi L, Torres R, Syed S, Boyle S, Ata A, Beyer T, Rosati C. Sharps and Needlestick Injuries Among Medical Students, Surgical Residents, Faculty, and Operating Room Staff at a Single Academic Institution.J Surgical Education 2017; 74(1):131-136.

24. Ceballos D, Marín D. Caracterización de los accidentes laborales en un hospital de alta complejidad de la región de Antioquia, Colombia. Revista Cubana de Salud y Trabajo 2015; 16(2):31-36.

25. Masoumi-Asl H, Rahbar M, Soltani A, Pezeshki Z, Khanaliha K, Kolifarhood G. Epidemiology of Needlestick Injuries Among Healthcare Workers in Tehran, Iran: A Cross-Sectional Study. Archives of Clinical Infectious Diseases 2016; 12(2):e37605.

26. United States of America. Needlestick Safety and Prevention Act of 2000. Public Law 2000; 6 November.

27. Agência Europeia para a Segurança e Saúde no trabalho. Directive 2010/32/EU - prevention from sharp injuries in the hospital and healthcare sector. Official J the European Union. Bruselas: CUE; 2010.

28. Brasil. Sistema de Informação de Agravos de Notificação (SINAN). SINAN-Acidentes com Material Biológico. Brasilia: SINAN; 2014.

29. Janjua NZ, Khan MI, Mahmood B. Sharp injuries and their determinants among health care workers at first level care facilities in Sindh Province, Pakistan. Tropical Medicine \& International Health 2010; 15(10):1244-1251.

30. Phipps W, Honghong W, Min Y, Burgess J, Pellico L, Watkins C, Guoping H, Williams A. Risk of medical sharps injuries among Chinese nurses. Am J Infect Control 2002; 30(5):277-282.

31. Garcia L, Facchini L. Exposures to blood and body fluids in Brazilian primary health care. Occupational Medicine 2009; 59(2):107-113. 
32. Oliveira A, Gonçalves J. Acidente ocupacional por material perfurocortante entre profissionais de saúde de um Centro Cirúrgico. Rev Esc Enferm USP 2010; 44(2):482-487.

33. Tapias-Vargas L, Torres-Bayona A, Vega-Vera A, Valencia-Ángel L, Orozco-Vargas L. Accidentes biológicos en médicos residentes de Bucaramanga, Colombia. Rev Colomb Cir 2010; 25(4):290-299.

34. Parsa-Pili J, Izadi N, Golbabaei F. Factors associated with needle stick and Sharp injuries among health care workers. International J Occupational Hygiene 2015; 5(4):191-197.

35. La-Rotta E, Garcia C, Barbosa F, Santos G, Vieira G, Carneiro M. Evaluation of the level of knowledge and compliance with standart precautions and the safety standard (NR-32) amongst physicians from a public university hospital, Brazil. Rev. Bras. Epidemiol 2013; 16(3):786-797.

36. Afridi A, Kumar A, Sayani R. Needle stick injuriesrisk and preventive factors: a study among health care workers in tertiary care hospitals in Pakistan. Global J Health Science 2013; 5(4):85-92.

37. Sharma R, Rasania S, Verma A, Singh S. Study of prevalence and response to needle stick injuries among health care workers in a tertiary care hospital in Delhi, India. Indian J Community Med 2010; 35(1):74-77.

38. Janjua N, Mahmood B, Khan M. Does knowledge about bloodborne pathogens influence the reuse of medical injection syringes among women in Pakistan? J Infect Public Health 2014; 7(4):345-355.

39. Moraes A, Rolim G, Costa A. O processo de adesão numa perspectiva analítico comportamental. Revista Brasileira de Terapia Comportamental e Cognitiva 2009; 11(2):329-345.

40. Bravoa B, Segura L, Postigoa J, Villafruelac J, Honrubiac V, Marchantec M. Hábitos, preferencias y satisfacción sexual en estudiantes universitarios. Revista Clínica de Medicina de Familia 2010; 3(3):150-157.

41. Rasmussen-Cruz B, Martín H, Alfaro-Alfaro N. Comportamientos de riesgo de ITS/SIDA en adolescentes trabajadores de hoteles de Puerto Vallarta y su asociación con el ambiente laboral. Salud Pública de México 2003; 45(1):s81-s91.

42. Kabir A, Tabatabaei S, Khaleghi S, Agah S, Kashani A, Moghimi M, Kerahroodi F, Alavian S, Alavian S. Knowledge, attitudes and practice of Iranian medical specialists regarding hepatitis B and C. Hepatitis monthly 2010; 10(3):176-182.

43. Garcia L, Facchini L. Vacinação contra a hepatite B entre trabalhadores da atenção básica à saúde Hepatitis $B$ vaccination among primary health care workers. Cad Saude Publica 2008; 24(5):1130-1140.

44. Phillips E, Conaway M, Jagger J. Percutaneous injuries before and after the Needlestick Safety and Prevention Act. N Eng J Med 2012; 366(7):670-667.

45. Florêncio V, Rodrigues C, Pereira M, Souza A. Adesão às precauções padrão entre os profissionais da equipe de resgate pré-hospitalar do Corpo de Bombeiros de Goias. Revista Eletrônica de Enfermagem 2003; 5(1):43-48.
46. Figueiredo R. Acidentes com risco biológico: a adesão de profissionais de saúde ao seguimento. Anais do $51^{\circ}$ Congresso Brasileiro de Enfermagem. Florianópolis: 358, 1999.

47. Hernández M. Health reform, equity, and the right to health in Colombia. Cad Saude Publica 2002; 18(4):991-1001.

48. Levino A, Carvalho E. Análise comparativa dos sistemas de saúde da tríplice fronteira: Brasil/Colômbia/ Peru. Revista Panamericana de Salud Pública 2011; 30(5):490-500.
Artigo apresentado em 30/03/2017

Aprovado em 03/07/2018

Versão final apresentada em 05/07/2018 
